\title{
Low-energy positron interactions with krypton
}

\author{
C. Makochekanwa, J. R. Machacek, A. C. L. Jones, P. Caradonna, D. S. Slaughter, R. P. McEachran, \\ J. P. Sullivan, and S. J. Buckman* \\ ARC Centre for Antimatter-Matter Studies, Research School of Physics and Engineering, Australian National University, Canberra, \\ ACT 0200, Australia
}

S. Bellm and B. Lohmann

ARC Centre for Antimatter-Matter Studies, The University of Adelaide, Adelaide, South Australia, Australia

D. V. Fursa and I. Bray

ARC Centre for Antimatter-Matter Studies, Curtin University of Technology, GPO Box U1987, Perth, Western Australia 6845, Australia

\author{
D.W. Mueller \\ Physics Department, University of North Texas, Denton, Texas 76201, USA \\ A. D. Stauffer \\ Department of Physics and Astronomy, York University, Toronto, Canada \\ M. Hoshino \\ Department of Physics, Sophia University, Chiyoda-ku, Tokyo, Japan
}

(Received 25 December 2010; published 30 March 2011)

\begin{abstract}
Cross sections for positron scattering from krypton have been measured with an energy resolution of $\sim 60 \mathrm{meV}$ over the energy range $0.5-60 \mathrm{eV}$. Absolute values of the grand total $\left(\sigma_{\mathrm{GT}}\right)$, positronium formation $\left(\sigma_{\mathrm{Ps}}\right)$, and grand total minus positronium formation $\left(\sigma_{\mathrm{GT}}-\sigma_{\mathrm{Ps}}\right.$ ) cross sections are presented. Theoretical estimations of $\sigma_{\mathrm{GT}}$ and $\sigma_{\mathrm{GT}}-\sigma_{\mathrm{Ps}}$ are also performed for this target using the convergent close-coupling method and the relativistic optical potential approach. We also provide experimental and theoretical results for elastic differential cross sections, for selected energies both below and above the Ps threshold. Where available, the present results are compared to both experimental and theoretical values from the literature.
\end{abstract}

DOI: 10.1103/PhysRevA.83.032721

PACS number(s): 34.80.Uv, 34.80.Bm, 36.10.Dr

\section{INTRODUCTION}

Since the early successes in the 1970s in producing lowenergy positron beams of high enough intensity for scattering experiments, experimental studies of positron scattering from noble gases have received the most attention. These experimental endeavors have been matched by the development of various theoretical approaches to estimate the measured cross sections. The grand-total cross section $\sigma_{\mathrm{GT}}$ (including elastic plus all energetically open inelastic channels) is the simplest to measure and has been the most studied. Among all the inelastic channels that contribute to $\sigma_{\mathrm{GT}}$, the cross section for the positronium (Ps) formation channel, $\sigma_{\mathrm{Ps}}$, is typically the largest in most atoms and molecules in this energy range. In many atomic and molecular systems, $\sigma_{\mathrm{Ps}}$ can constitute up to half of $\sigma_{\mathrm{GT}}$, particularly in the region within 10-20 eV above the Ps threshold. The strength and nature of this inelastic scattering channel also provides a strict test for scattering theory, as it must be included explicitly in any "complete" theoretical investigation.

Ps formation is also important at energies below several hundred $\mathrm{eV}$ as it is the main channel leading to annihilation and production of $511 \mathrm{keV}$ gamma rays. The detection of these gamma rays is important in applications of positron

\footnotetext{
*Stephen.Buckman@anu.edu.au
}

physics and chemistry to areas such as positron emission tomography and positron annihilation lifetime spectroscopy. An understanding of Ps formation, both qualitative and quantitative, for the rare gases is thus an opportunity to benchmark experimental and theoretical techniques for atomic systems that are experimentally and theoretically tractable, although the latter poses a significant challenge. Techniques that are established with the noble gases can then be applied to the more significant challenges that are presented by interactions with large molecules of biological interest, for example.

Most of the available experimental values for $\sigma_{\mathrm{GT}}$ for krypton (Kr) are from the 1970s and 1980s. This earlier work and the theoretical calculations for $\mathrm{Kr}$ up until 1992 have been well summarized by Baluja and Jain [1]. Thereafter, the only work on $\sigma_{\mathrm{GT}}$ has been a more recent theoretical calculation by Parcell et al. [2]. A number of experimental and theoretical reports have been made for studies of Ps formation from the noble gases, including $\mathrm{Kr}$. The experimental results for Ps formation cross sections $\sigma_{\mathrm{Ps}}$ from $\mathrm{Kr}$ have been well summarized by Marler et al. [3], while the current state of the theoretical calculations for this cross section is represented by the most recent publication of Gilmore et al. [4].

In addition to the above studies, other relevant studies for positron scattering from $\mathrm{Kr}$ exist in the literature and are summarized in the following few references on elastic differential, electronic excitation, and ionization cross 
sections. Data for elastic differential cross sections consist of one absolute [5] and one relative [6] measurement, and a number of theoretical estimations; see, for example, Refs. [7,8]. It is worth noting that even with the success in carrying out absolute measurements [5], experimental data were reported at only two energies, 1 and $2 \mathrm{eV}$. Electronic excitation cross sections have only been reported theoretically [9], while ionization cross sections have been studied both experimentally $[3,10]$ and theoretically $[11,12]$.

The most notable observation from a perusal of the literature results for both $\sigma_{\mathrm{GT}}$ and $\sigma_{\mathrm{Ps}}$ is that the level of agreement among them is poor $[3,13]$. Thus, part of the motivation for this study was to provide accurate, high-resolution measurements for this noble-gas target, combined with the latest theoretical treatments. The present work also represents an important study of absolute elastic differential cross sections. In the following sections we briefly discuss the experimental techniques and theoretical approaches that have been applied in the present work and present results for total, total minus positronium formation, elastic differential, and positronium formation cross sections. These are compared with values from the literature.

\section{EXPERIMENTAL PROCEDURE}

The measurements presented here were carried out on the low-energy positron beamline facility at the Australian National University. The experimental apparatus is based on the "Surko" trap system, developed at University of California San Diego (UCSD) [14,15], and has been comprehensively described elsewhere [16], so only a brief overview of the operation will be presented here. Positrons are obtained from a radioactive source of ${ }^{22} \mathrm{Na}$ that had an activity of approximately $40 \mathrm{mCi}$ for the measurements presented in this paper. A solid neon moderator is used to form a low-energy positron beam, which is radially confined using solenoidal magnetic fields $(100 \mathrm{G})$ and transported into a three-stage buffer-gas trap where the field is $530 \mathrm{G}$. The trap electrodes form a stepped electrostatic potential well, and positrons lose energy inside the trap through inelastic collisions with a mixture of $\mathrm{N}_{2}$ and $\mathrm{CF}_{4}$ buffer gases, in the process thermalizing to room temperature. This trapped cloud of positrons becomes the reservoir for a pulsed positron beam. Trap operation is typically cycled at approximately $100-200 \mathrm{~Hz}$ with up to $1000-4000$ positrons per pulse. Careful control over the beam formation means that the energy width of the beam is comparable to the temperature of the trapped positron cloud. In these experiments, the typical energy resolution was $50-70 \mathrm{meV}$, while typical target pressures were about 0.25 mTorr.

The positron beam is directed to a gas cell of length $100 \mathrm{~mm}$, which is constructed from gold-plated copper and which contains the $\mathrm{Kr}$ target gas. The gas cell entrance and exit apertures are $5 \mathrm{~mm}$ in diameter. The potential of the gas cell defines the energy of the positrons within the cell, and the target gas is localized to the $100-\mathrm{mm}$ path length. Target density inside the cell is maintained so that total positron scattering is no more than $10 \%$ of the unscattered beam in order to avoid multiple-scattering effects. With the exception of those positrons that form Ps and annihilate in the cell, almost all of the positrons are transmitted through the gas cell and pass through a retarding potential analyzer (RPA), which is sensitive only to the parallel energy component of the beam $E_{\|}$. The limitation of this technique in failing to detect positrons scattered in a small region around $90^{\circ}$ is discussed in Sec. II B. Those that are transmitted by the RPA then encounter a double-stack, microchannel-plate detector. In a collision with a target gas atom, the positron can be scattered through some angle $\theta$, in the process losing some $E_{\|}$, and it can also lose some of its total energy if inelastic processes are energetically allowed [17]. Ps formation is also possible above the Ps formation threshold $E_{\mathrm{Ps}}$, corresponding to $7.200 \mathrm{eV}$ for $\mathrm{Kr}$, and results directly in a loss of positrons from the beam.

In our experimental approach, for both the total and elastic differential cross sections, the zero for the energy scale is established with a retarding potential analysis of the beam, i.e., with the energy scale defined relative to the "cutoff" position of the beam. This procedure is estimated to result in an uncertainty of $\pm 20 \mathrm{meV}$ in the energy scale. The scattering cell target pressure was measured using a Model 690 MKS Baratron capacitance manometer with a full range of 1 Torr and a stated measurement accuracy of $\pm 0.1 \%$. There are a number of possible sources of systematic error in our measurements, and these have been estimated and accounted for, where possible, and have been discussed previously [18]. Statistical errors typically vary between $1 \%$ and $5 \%$ for the total scattering and Ps formation measurements, while for the differential elastic measurements they can be significantly larger under some circumstances $(>10 \%)$. Systematic effects typically account for an uncertainty of less than $5 \%$. Thus, the uncertainty in the total and positronium formation cross sections are in the range $5 \%-7 \%$, while those for the differential cross section (DCS) measurements are typically between $7 \%$ and $15 \%$, although in some cases they are significantly larger.

\section{A. Total cross sections}

The techniques that have been used for the measurements of total scattering and total Ps formation cross sections have been presented in a recent paper [18] and previous work [17] and will not be repeated in detail here. The technique involves the measurement of a number of transmitted positron intensities at various values of the potential applied to the RPA. From these intensities and the measured gas pressure and scattering cell length, $\sigma_{\mathrm{GT}}, \sigma_{\mathrm{Ps}}$, and $\sigma_{\mathrm{GT}}-\sigma_{\mathrm{Ps}}$ can be directly determined using the Beer-Lambert attenuation law [18]. The path length through the target gas was taken as the geometrical length of the scattering cell. It was experimentally established to be so by measurement of the benchmark total cross sections for He [16,19].

As with any linear-transmission type of experiment using a scattering cell, our experimental technique inevitably has some angular discrimination limitations. This arises from the inability to distinguish between positrons that are elastically scattered at small angles from those in the primary, unscattered beam. This issue results in the directly measured cross section being somewhat smaller than the "true" value. The extent of the problem depends on the angular discrimination of the apparatus and the nature of the differential elastic scattering 
cross section in this forward angle region. It has been discussed in detail in a recent publication [20].

We can, however, make a reasonably accurate estimate of the contribution to the total cross section that is missing from our measured cross section if we have knowledge of our angular discrimination and of the differential elastic scattering cross section. Our technique, described in detail in [18], relies on measuring the transmitted positron intensity at the beam cutoff point on the retarding potential analyzer voltage axis (see Refs. [17,18] for full details on this technique). In practice, however, it is not possible to make a measurement of the intensity at exactly the cutoff voltage because this also coincides with the cutoff point of the primary positron beam. To avoid effects associated with the cutoff, the positron intensity must be measured at a retarding potential voltage sufficiently offset from the cutoff. An offset of $\Delta V=-100 \mathrm{mV}$ (i.e., $>3$ standard deviations) away from the cutoff was used for these measurements. This restriction means that the measured total cross section excludes some contribution from the DCS corresponding to this inaccessible angular range.

The missing angular ranges $\left(0 \pm \theta_{\min }\right)^{\circ}$ and $\left(180 \pm \theta_{\min }\right)^{\circ}$ can be calculated using a method discussed previously $[17,18]$, where the critical angle $\theta_{\min }$ is given by

$$
\theta_{\min }=\sin ^{-1} \sqrt{\frac{e \Delta V}{E_{\mathrm{SC}}}}
$$

where $E_{\mathrm{SC}}$ is the positron energy in $\mathrm{eV}$ and $e$ is the elementary charge. The results for this calculation and the resulting predicted corrections for the total cross section are given in Table I. Here we have used the differential cross sections calculated from the present relativistic optical potential (ROP) calculation to estimate the correction.

It is worth noting that the forward-scattering effects are due to positrons elastically scattered into the experimentally inaccessible angular range $0 \pm \theta_{\min }$. Therefore, this only affects the elastic-scattering cross section, and thus, the correction needs only to be applied for the measured $\sigma_{\mathrm{GT}}$ and $\sigma_{\mathrm{GT}}-\sigma_{\mathrm{Ps}}$ and not for $\sigma_{\mathrm{Ps}}$.

\section{B. Elastic differential cross sections}

By making use of principles of positron motion and scattering in a strong magnetic field, the same experimental apparatus and techniques that are used for the total crosssection measurements can be used for differential cross-section determinations. The details on how to measure and analyze the data for elastic differential cross sections have been

TABLE I. Estimated missing angular range $0 \pm \theta_{\min }$ and the corresponding $\sigma_{\mathrm{GT}}$ and $\sigma_{\mathrm{GT}}-\sigma_{\mathrm{Ps}}$ corrections for $\mathrm{Kr}$ for selected positron scattering energies.

\begin{tabular}{lcc}
\hline \hline Energy $(\mathrm{eV})$ & $\theta_{\min }$ & Correction $(\%)$ \\
\hline 0.5 & 27 & 19 \\
1.0 & 18 & 17 \\
2.0 & 13 & 13 \\
5.0 & 8 & 8 \\
8.0 & 6 & 5 \\
\hline \hline
\end{tabular}

discussed elsewhere [17,21] and thus will only be summarized briefly here. As highlighted above, the RPA analyses only the parallel energy component of the positron beam, $E_{\|}$. The transmitted current, $I$, as a function of $E_{\|}$can be related to the differential scattering cross section (below and above any inelastic thresholds) through the relationship

$$
\frac{d \sigma}{d \Omega}=\frac{1}{\pi n l I_{0}}\left(\frac{d I_{C}(E)}{d E_{\|}}\right)
$$

where $n$ is the number density of the target gas in the scattering cell, $l$ is the length of the scattering cell, $I_{0}$ is the incident positron intensity, and $E$ is the scattering energy. All positrons that are scattered in the cell travel through to the RPA and detector. Positrons scattered in the backward direction (i.e., through angles greater than $90^{\circ}$ ) are reflected from the potential barriers associated with the trap and other elements and can travel back through the gas cell. As with the total cross-section measurements, the scattering probability on a single pass through the cell must be adjusted so that there is a small chance of rescattering. That is, the total scattering probabilities for these measurements are also kept to less than $10 \%$. The result of this backward-scattered contribution is that the measured cross section at any angle $\theta$ actually consists of contributions from scattering through $\theta$ and $180-\theta$; that is, the cross section is "folded" around $90^{\circ}$ [17].

Positrons scattered near $90^{\circ}$ have a greatly increased path length inside the gas cell after the scattering event, and have a higher probability of rescattering or being lost from the beam, as has been discussed in detail in [17]. In addition, as also discussed in [17], the $0^{\circ}$ of the scattering angle range corresponds to the RPA cutoff voltage point and, as highlighted above in the discussion for total cross sections, it is not possible to make a measurement at exactly the cutoff voltage. For this reason, the positron current must be measured at a retarding potential voltage sufficiently offset from the cutoff point. Therefore, the angular range that can be measured by this technique is limited to $\theta_{\min }<\theta<90^{\circ}$.

In this paper we also present measurements of elastic DCS at energies above the first inelastic excitation threshold, and this requires an alteration of the experimental technique from that described above for the case of pure elastic scattering. This is a result of the need to separate those positrons scattered elastically from those that have undergone inelastic scattering. Details of the technique that we use for achieving this have been described for measurements of electronic excitation cross sections [22], and also in [17], and are only briefly summarized here.

The lowest inelastic threshold, Ps formation, is a loss process whereby the positron that has formed Ps is not detected and thus is easily excluded from the data analysis. This leaves electronic excitations and ionization as the inelastic channels that need to be considered in the above-threshold elastic DCS measurements. The technique used to distinguish between these different scattering channels is directly connected to the motion of positrons in a magnetic field. The total positron energy can be expressed in terms of the components that are parallel and perpendicular to the magnetic field, $E_{\text {tot }}=E_{\|}+$ $E_{\perp}$. However, at energies above the first electronic excitation, 
there is no longer a one-to-one relationship between $E_{\|}$and $\theta$ for a unique elastic DCS solution for Eq. (2) due to energy loss from $E_{||}$resulting from excitation plus angular scattering involving an atomic excited state.

For a slowly varying magnetic field, the magnetic moment of a charged particle $E_{\perp} / B$ is an adiabatic invariant. As a result, by changing the ratio of the field in the scattering cell to that at the RPA, often referred to as the "beach ratio," $M=B_{\mathrm{SC}} / B_{\mathrm{RPA}}$, where $B_{\mathrm{SC}}$ and $B_{\mathrm{RPA}}$ are the values of the magnetic field at the scattering cell and RPA, respectively; this invariant quantity can be used to our advantage. Depending on the magnitude of $M$, this technique enables us to convert some, or all, of the positron's perpendicular energy back into the parallel component. In this way we can separate losses of $E_{\|}$that are due to various, discrete inelastic scattering events from those losses that are due to the transfer of energy from $E_{\|}$ to $E_{\perp}$ as a result of angular elastic scattering. For a given $E_{\text {tot }}$, an $M$ value can be selected that allows for this to be achieved effectively, with a slight reduction in the measured angular range being the only significant consequence. For the present measurements of above-threshold elastic scattering we used $M=3$.

\section{THEORETICAL APPROACHES}

We employ two theoretical treatments for computing cross sections for positron scattering from $\mathrm{Kr}$. One is the relativistic optical potential (ROP) calculation [23], which has been used to calculate the grand total cross section $\sigma_{\mathrm{GT}}$ below $E_{\mathrm{Ps}}$ and the grand total minus Ps formation $\left(\sigma_{\mathrm{GT}}-\sigma_{\mathrm{Ps}}\right)$ above $E_{\mathrm{Ps}}$. The other approach is the convergent close-coupling (CCC) approach [24] and is a calculation of $\sigma_{\mathrm{GT}}$ at all energies. Both techniques have been discussed in detail recently [18], and only a brief description is given here.

\section{A. The relativistic optical potential method}

The theoretical cross sections presented here for krypton were determined from the solution of the relativistic DiracFock scattering equations containing a static, a polarization, and an $a b$ initio complex optical potential. The details of this method have been given previously in [23], and its application to positron scattering from neon and argon is discussed in [18]. Thus, only a brief discussion of its application to krypton is given here.

In the case of krypton, the dipole and the next three higher multipoles were included in the polarization potential. Whenever the energy of the incident positron was such that inelastic channels (excitation and ionization) were open, the imaginary part of the optical potential was incorporated to describe absorption processes. This absorption potential is both nonlocal and $a b$ initio and is determined as an expansion over the inelastic channels of the target, which include both the excitation of the discrete bound states as well as single ionization of the target.

The discrete Dirac-Fock wave functions of the target were determined using the multiconfiguration Dirac-Fock (MCDF) program of Grant et al. [25]. Here the absorption potential included the following 26 excited states (in intermediate coupling notation):

$$
\begin{array}{llll}
4 \bar{d}[1 / 2]_{J=1}^{\mathrm{o}} & 4 d[3 / 2]_{J=1}^{\mathrm{o}} & 4 \bar{d}[3 / 2]_{J=1}^{\mathrm{o}} & 4 d[7 / 2]_{J=3}^{\mathrm{o}} \\
4 \bar{d}[5 / 2]_{J=3}^{\mathrm{o}} & 4 d[5 / 2]_{J=3}^{\mathrm{o}} & 5 s[3 / 2]_{J=1}^{\mathrm{o}} & 5 s[1 / 2]_{J=1}^{\mathrm{o}} \\
5 p[1 / 2]_{J=0} & 5 \bar{p}[1 / 2]_{J=0} & 5 p[5 / 2]_{J=2} & 5 \bar{p}[3 / 2]_{J=2} \\
5 p[3 / 2]_{J=2} & 5 \bar{d}[1 / 2]_{J=1}^{\mathrm{o}} & 5 d[3 / 2]_{J=1}^{\mathrm{o}} & 5 \bar{d}[3 / 2]_{J=1}^{\mathrm{o}} \\
5 d[7 / 2]_{J=3}^{\mathrm{o}} & 5 \bar{d}[5 / 2]_{J=3}^{\mathrm{o}} & 5 d[5 / 2]_{J=3}^{\mathrm{o}} & 6 s[3 / 2]_{J=1}^{\mathrm{o}} \\
6 s[1 / 2]_{J=1}^{\mathrm{o}} & 6 p[1 / 2]_{J=0} & 6 \bar{p}[1 / 2]_{J=0} & 6 \bar{p}[5 / 2]_{J=2} \\
6 \bar{p}[3 / 2]_{J=2} & 6 p[3 / 2]_{J=2} & &
\end{array}
$$

Here $J$ represents the total angular momentum of the atom, $\bar{p}$ represents a $p$ orbital with $j=1 / 2$, and $p$ represents a $p$ orbital with $j=3 / 2$. Similarly, $\bar{d}$ and $d$ represent $d$ orbitals with $j=3 / 2$ and $5 / 2$, respectively. In addition, the absorption potential included the following continuum states:

$$
\begin{array}{llll}
\varepsilon s[3 / 2]_{J=1}^{\mathrm{o}} & \varepsilon s[1 / 2]_{J=1}^{\mathrm{o}} & \varepsilon p[1 / 2]_{J=0} & \varepsilon \bar{p}[1 / 2]_{J=0} \\
\varepsilon p[5 / 2]_{J=2} & \varepsilon p[3 / 2]_{J=2} & \varepsilon \bar{p}[3 / 2]_{J=2} & \varepsilon \bar{d}[1 / 2]_{J=1}^{\mathrm{o}} \\
\varepsilon d[3 / 2]_{J=1}^{\mathrm{o}} & \varepsilon \bar{d}[3 / 2]_{J=1}^{\mathrm{o}} & \varepsilon d[7 / 2]_{J=3}^{\mathrm{o}} & \varepsilon d[5 / 2]_{J=3}^{\mathrm{o}} \\
\varepsilon \bar{d}[5 / 2]_{J=3}^{\mathrm{o}} & \varepsilon s[1 / 2]_{J=0} & \varepsilon p[3 / 2]_{J=1}^{\mathrm{o}} & \varepsilon \bar{p}[1 / 2]_{J=1}^{\mathrm{o}} \\
\varepsilon d[5 / 2]_{J=2} & \varepsilon \bar{d}[3 / 2]_{J=2} & &
\end{array}
$$

where $\varepsilon$ represents the energy of the ejected electron, originally either a bound $4 \bar{p}$ or $4 p$ electron and, above $27.5 \mathrm{eV}$, a $4 s$ electron.

Above the inelastic threshold, the scattering phase shifts are complex, i.e.,

$$
\eta_{l}^{ \pm}=\delta_{l}^{ \pm}+i \gamma_{l}^{ \pm}
$$

where the $+(-)$ sign refers to a spin-up (spin-down) positron. The elastic and inelastic cross sections are then determined from these phase shifts as described in [23].

\section{B. The convergent close-coupling method}

The CCC method solves the nonrelativistic Schrödinger equation for the positron-krypton scattering system by performing a close-coupling expansion of the total wave function in a basis of $\mathrm{Kr}$ atom target states. The resulting set of close-coupling equations is converted to coupled LippmannSchwinger equations for the $T$ matrix that are solved by standard techniques [26]. The target-state basis is obtained by diagonalization of the target Hamiltonian in a basis constructed from Sturmian (Laguerre) one-electron functions. The resulting basis provides a square-integrable representation for the target bound states and the continuum. The single-centered target-state expansion used in the CCC method does not explicitly include positronium formation channels. However, outside the energy region between the Ps formation and directionization thresholds, they can be adequately represented due to completeness of the basis [18,27,28].

Calculation of $\mathrm{Kr}$ target states proceeds along the same scheme as used recently for $\mathrm{Ne}$ and $\mathrm{Ar}$ [18]. First, we perform self-consistent Hartree-Fock calculations for the $\mathrm{Kr}^{+}[\mathrm{Zn}] 4 p^{5}$ ground state. Then, we conduct configuration interaction (CI) 
calculations by diagonalizing the $\mathrm{Kr}$ Hamiltonian in the basis of all possible [ $\mathrm{Zn}] 4 p^{5} \mathrm{nl}$ configurations. The active electron is represented by the Laguerre basis with basis size $N_{l}=20-l$ and exponential falloff $\lambda_{l}=2.5$ for $l \leqslant l_{\max }=8$. These lead to 377 states in the close-coupling expansion. The accuracy of the present $\mathrm{Kr}$ structure model can be assessed by comparing the calculated ground state ionization energy, $13.38 \mathrm{eV}$, with the experimental value of $13.99 \mathrm{eV}$.

The total cross section calculated in the CCC method is affected by the value of the static dipole polarizability of the $\mathrm{Kr}$ atom. We have used a semiempirical two-electron polarization potential [29] in order to bring the calculated static dipole polarizability $\alpha$ of 17.26 a.u. close to its experimental value of 16.8 a.u. [30]. This potential modifies the dipole term of the positron-electron Coulomb potential and is given by the following expression:

$$
\begin{aligned}
V_{p}\left(r_{0}, r_{i}\right)= & -\frac{\alpha}{r_{0}^{3} r_{i}^{3}}\left(\mathbf{r}_{0} \cdot \mathbf{r}_{i}\right) \\
& \times \sqrt{\left(1-e^{-\left(r_{0} / \rho\right)^{6}}\right)\left(1-e^{-\left(r_{i} / \rho\right)^{6}}\right)}
\end{aligned}
$$

where the index 0 refers to the positron and the index $i$ refers to one of the krypton $4 p^{6}$ electrons. The parameters of the twoelectron polarization potential are chosen to be $\alpha=10.0$ a.u. and $\rho=4.5$ a.u.

Finally, we note that krypton is a sufficiently heavy atom for which a relativistic approach is preferable. This is particularly important for excited states of krypton. However, for the elastic scattering cross section and aggregate observables such as the total cross section, the use of a nonrelativistic approach is likely to be sufficient.

\section{RESULTS AND DISCUSSION}

In the discussion that follows we present the various total cross section $\left(\sigma_{\mathrm{GT}}, \sigma_{\mathrm{Ps}}\right.$, and $\left.\sigma_{\mathrm{GT}}-\sigma_{\mathrm{Ps}}\right)$ results and compare them with data from the literature. These data have been corrected for the angular discrimination limitations discussed earlier in Sec. II, using our elastic differential cross sections calculated with the ROP method. Following this, we then discuss the results and calculations for the elastic differential cross sections and compare these with literature results, where available.

\section{A. Total cross sections}

The experimental results for $\sigma_{\mathrm{GT}}, \sigma_{\mathrm{Ps}}$, and $\sigma_{\mathrm{GT}}-\sigma_{\mathrm{Ps}}$ are presented in Table II, and both experimental values and the current calculations are shown in Figs. 1 and 2.

\section{Grand total cross section $\sigma_{\mathrm{GT}}$}

Figure 1(a) shows the current experimental and the CCC theoretical results for $\sigma_{\mathrm{GT}}$ and results from the literature. The present experimental result can be compared here with five other measurements [13,31-34], and they all largely agree with the present result with respect to the overall energy dependence of the cross section. The principal features are strong scattering at low energies $\left(\sim 12 \AA^{2}\right.$ at $\left.0.5 \mathrm{eV}\right)$, which drops away smoothly until the Ps formation threshold $\left(\sim 7 \AA^{2}\right.$ at $\left.7.2 \mathrm{eV}\right)$, above which the total cross section rises until $10 \mathrm{eV}$, where it plateaus at a level of $11-12 \AA^{2}$ out to $60 \mathrm{eV}$. The one exception to this trend is the data of [31], which decrease steadily in magnitude below $7 \mathrm{eV}$. Below $7.2 \mathrm{eV}$ the scattering is purely elastic, and the large low-energy cross section is driven mainly by polarization effects, the dipole polarizability of $\mathrm{Kr}$ being quite large at 16.8 a.u. The rapid rise above $7.2 \mathrm{eV}$ is due to the opening up of the Ps formation channel and is characteristic of the behavior in other atoms and molecules when this strong-scattering channel is opened.

The recent experimental results of Jay and Coleman [32] agree well with the earlier results of Dababneh et al. [33] in magnitude over the whole energy of overlap. The current results agree well with the results of Sinapius et al. [13] below $5 \mathrm{eV}$ and are larger than those of Dababneh et al. over all the energy range of overlap; e.g. they are $\sim 50 \%$ higher between 1 and $7 \mathrm{eV}$ and $\sim 20 \%$ higher at $10 \mathrm{eV}$, with the difference decreasing with impact energy down to about $8 \%$ at $50 \mathrm{eV}$. In general, the present results are higher than all others at most energies below the Ps threshold, and we have demonstrated in a previous publication [20] that this is due, in large part, to differences in the degree of discrimination in the various experiments against forward elastic scattering. Poor angular discrimination in the forward direction results in a smaller total scattering cross section due to the exclusion of regions of the angular scattering intensity. For the heavier rare gases this can be particularly important as the elastic DCS are significantly forward peaked at low energies as a result of the large dipole polarizabilities. Thus, we believe these differences result from the different levels of angular discrimination in the various experiments, and a detailed discussion can be found in [20].

Perhaps the best agreement for $\sigma_{\mathrm{GT}}$ is found between the present results and the recent preliminary measurements of the Trento group [34]. These latter measurements show essentially the same energy dependence of the present results but lie $\sim 5 \%-10 \%$ below in magnitude. Given that they are not corrected for forward-angle-scattering effects, and that the angular discrimination of their experiment, based on the physical size of the scattering cell apertures, is $4^{\circ}$ [35], the application of such a correction would remove most, but not all, of this discrepancy with the present results. These authors also estimate that when the motion of the positrons in their relatively weak magnetic field is taken into account, the estimated angular discrimination may be significantly larger.

Good agreement is observed between the present experimental result and the present CCC calculation over the whole energy range, with the exception of the region between $E_{\mathrm{Ps}}$ $(7.2 \mathrm{eV})$ and the ionization threshold $E_{\text {ion }}$ at $14 \mathrm{eV}$. In this region we do not present calculated values from the $\mathrm{CCC}$ approach but merely join the value of the cross section at $E_{\mathrm{Ps}}$ with that at $E_{\text {ion }}$ with a straight line to guide the eye. This issue highlights one of the problems that theory has in handling Ps formation in this energy region. In this region the presence of the Ps formation channel, leading also to ionization, requires a two-center expansion for accurate calculations. This problem has now been solved by new CCC formulations for helium [24], and work is currently ongoing to extend the two-center technique to the heavier noble gases. Good agreement between the present experimental results and the earlier theoretical results of Baluja and Jain [1] is noted above $30 \mathrm{eV}$. However, their calculated cross section differs from both our experimental and calculated cross sections, as it rather 
TABLE II. Absolute grand total $\left(\sigma_{\mathrm{GT}}\right)$, grand total minus positronium formation $\left(\sigma_{\mathrm{GT}}-\sigma_{\mathrm{Ps}}\right)$, and positronium formation $\left(\sigma_{\mathrm{Ps}}\right)$ cross sections for krypton in units of $10^{-16} \mathrm{~cm}^{2}$. Energy is in units of $\mathrm{eV}$. Experimental uncertainties are explained in the text.

\begin{tabular}{|c|c|c|c|}
\hline Energy & $\sigma_{\mathrm{GT}}$ & $\sigma_{\mathrm{GT}}-\sigma_{\mathrm{Ps}}$ & $\sigma_{\mathrm{Ps}}$ \\
\hline 0.500 & 11.950 & 11.950 & $\ldots$ \\
\hline 1.000 & 10.509 & 10.599 & $\ldots$ \\
\hline 1.500 & 8.965 & 8.991 & $\ldots$ \\
\hline 2.000 & 8.760 & 8.837 & $\ldots$ \\
\hline 2.500 & 7.949 & 8.071 & $\ldots$ \\
\hline 3.000 & 7.861 & 7.884 & $\ldots$ \\
\hline 3.500 & 7.598 & 7.611 & $\ldots$ \\
\hline 4.000 & 7.431 & 7.487 & $\ldots$ \\
\hline 4.500 & 7.287 & 7.197 & $\ldots$ \\
\hline 5.000 & 7.096 & 7.088 & $\ldots$ \\
\hline 5.500 & 6.859 & 6.841 & $\ldots$ \\
\hline 6.000 & 7.003 & 6.939 & $\ldots$ \\
\hline 6.500 & 6.910 & 6.836 & $\ldots$ \\
\hline 7.000 & 7.116 & 6.958 & 0.158 \\
\hline 7.500 & 7.906 & 6.987 & 0.919 \\
\hline 8.000 & 8.533 & 6.792 & 1.741 \\
\hline 8.500 & 9.000 & 6.552 & 2.448 \\
\hline 9.000 & 9.412 & 6.340 & 3.072 \\
\hline 9.500 & 9.490 & 6.122 & 3.368 \\
\hline 10.000 & 9.909 & 5.938 & 3.971 \\
\hline 11.000 & 10.718 & 6.182 & 4.535 \\
\hline 12.000 & 10.870 & 5.999 & 4.871 \\
\hline 13.000 & 11.053 & 5.807 & 5.246 \\
\hline 14.000 & 11.101 & 5.765 & 5.336 \\
\hline 15.000 & 11.177 & 5.971 & 5.206 \\
\hline 16.000 & 11.159 & 6.041 & 5.118 \\
\hline 17.000 & 11.575 & 6.322 & 5.253 \\
\hline 18.000 & 11.474 & 6.496 & 4.977 \\
\hline 19.000 & 11.715 & 6.654 & 5.061 \\
\hline 20.000 & 11.746 & 6.861 & 4.885 \\
\hline 21.000 & 11.325 & 6.482 & 4.608 \\
\hline 22.000 & 11.533 & 6.664 & 4.644 \\
\hline 23.000 & 11.939 & 6.964 & 4.771 \\
\hline 24.000 & 11.507 & 7.059 & 4.402 \\
\hline 25.000 & 11.964 & 7.456 & 4.365 \\
\hline 26.000 & 11.903 & 7.252 & 4.487 \\
\hline 27.000 & 11.843 & 7.231 & 4.476 \\
\hline 28.000 & 11.578 & 7.395 & 4.118 \\
\hline 29.000 & 11.924 & 7.552 & 4.303 \\
\hline 30.000 & 12.084 & 7.676 & 4.282 \\
\hline 31.000 & 11.778 & 7.762 & 3.957 \\
\hline 32.000 & 11.513 & 7.856 & 3.578 \\
\hline 33.000 & 11.884 & 7.944 & 3.793 \\
\hline 34.000 & 11.604 & 7.935 & 3.564 \\
\hline 35.000 & 12.081 & 8.212 & 3.663 \\
\hline 36.000 & 11.978 & 8.074 & 3.705 \\
\hline 37.000 & 11.593 & 8.207 & 3.338 \\
\hline 38.000 & 11.754 & 8.275 & 3.348 \\
\hline 39.000 & 11.737 & 8.399 & 3.188 \\
\hline 40.000 & 11.693 & 8.389 & 3.159 \\
\hline 41.000 & 11.639 & 8.269 & 3.240 \\
\hline 42.000 & 11.363 & 8.316 & 2.872 \\
\hline 43.000 & 11.566 & 8.459 & 3.048 \\
\hline 44.000 & 11.264 & 8.309 & 2.742 \\
\hline 45.000 & 11.731 & 8.508 & 3.040 \\
\hline 46.000 & 11.473 & 8.549 & 2.662 \\
\hline
\end{tabular}

TABLE II. (Continued.)

\begin{tabular}{llll}
\hline \hline Energy & $\sigma_{\mathrm{GT}}$ & $\sigma_{\mathrm{GT}}-\sigma_{\mathrm{Ps}}$ & $\sigma_{\mathrm{Ps}}$ \\
\hline 47.000 & 11.599 & 8.563 & 2.895 \\
48.000 & 10.936 & 8.567 & 2.244 \\
49.000 & 11.376 & 8.728 & 2.567 \\
50.000 & 11.132 & 8.744 & 2.212 \\
51.000 & 11.114 & 8.506 & 2.537 \\
52.000 & 11.314 & 8.898 & 2.284 \\
53.000 & 11.044 & 8.810 & 2.174 \\
54.000 & 11.371 & 8.974 & 2.152 \\
55.000 & 11.041 & 9.007 & 1.887 \\
56.000 & 10.765 & 8.993 & 1.714 \\
57.000 & 10.866 & 8.722 & 1.918 \\
58.000 & 10.681 & 9.023 & 1.466 \\
59.000 & 10.933 & 9.194 & 1.557 \\
60.000 & 11.104 & 9.298 & 1.537 \\
\hline \hline
\end{tabular}

rapidly decreases with decreasing energy below $30 \mathrm{eV}$. With the exception of a similar energy dependence below about $12 \mathrm{eV}$, the present cross section is neither in good qualitative nor quantitative agreement with the result of Parcell et al. [2], who did not explicitly calculate the total cross section, but rather derived it semi-analytically. They combined their calculated elastic integral cross section with partial crosssection results from different sources in the literature: the Ps formation cross section from McAlinden and Walters [36] and ionization cross sections of Campeanu et al. [37-39]. However, as displayed in Fig. 2(b), the results of [36] fall significantly below the present measurements over much of the energy range, while the recent ROP calculations of the ionization cross section in [12] are considerably larger than those of Campeanu et al. Thus, the difference between the present measurements and the cross sections calculated in [2] may largely be due to the Ps formation and ionization cross sections used in the latter.

\section{Grand total minus $P$ s formation cross section, $\sigma_{\mathrm{GT}}-\sigma_{\mathrm{Ps}}$}

Figure 1(b) shows the current experimental and theoretical ROP results for $\sigma_{\mathrm{GT}}-\sigma_{\mathrm{Ps}}$, compared with other results from the literature. Below the first excited state threshold for $\mathrm{Kr}$ ( $10.03 \mathrm{eV}$ for positron impact) this channel is equivalent to the total elastic scattering channel. One reason for measuring this cross section is that it may be sensitive to channel-coupling effects, which are possible between the elastic scattering channel and each newly opening inelastic channel. Such effects might be expected at or near the thresholds for Ps formation, electronic excitation, and ionization. This has been the subject of a recent detailed study by our laboratory [40]. In this work strong channel coupling, manifesting as Wigner cusps, was observed in all of the rare gases at the Ps threshold. This is clearly seen in $\sigma_{\mathrm{GT}}-\sigma_{\mathrm{Ps}}$ at $E_{\mathrm{Ps}}$ in Fig. 1(b) but is not seen in any of the other results shown. A difference is clearly observed with the only other experimental result, that of Jay and Coleman [32]. They observe a rise in $\sigma_{\mathrm{GT}}-\sigma_{\mathrm{Ps}}$ above the Ps threshold, but the energy dependence and magnitude of their result is quite different to the present work. This cusp feature is also not observed in the current ROP calculation since the ROP approach does not explicitly treat Ps formation. In addition, 

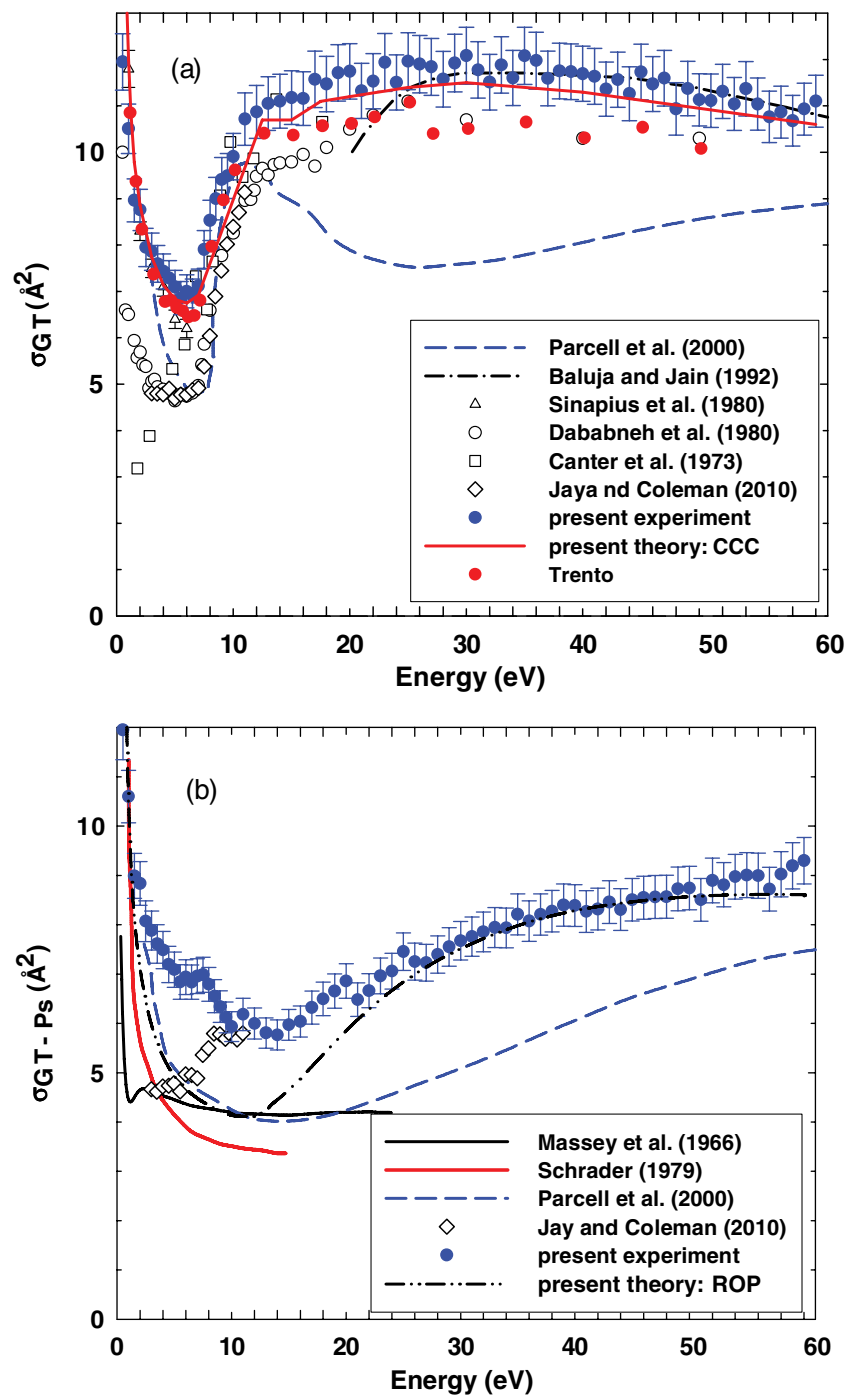

FIG. 1. (Color online) Present positron scattering from $\mathrm{Kr}$ (a) absolute total cross sections $\sigma_{\mathrm{GT}}$, in units of $10^{-16} \mathrm{~cm}^{2}$, in comparison with literature results from Parcell et al. [2], Baluja and Jain [1], Sinapius et al. [13], Dababneh et al. [33], Canter et al. [31], Jay and Coleman [32], and Trento [34] and (b) absolute total minus positronium formation cross sections, $\sigma_{\mathrm{GT}}-\sigma_{\mathrm{Ps}}$, in units of $10^{-16} \mathrm{~cm}^{2}$, in comparison with literature values from Massey et al. [42], Schrader [41], Parcell et al. [2], and Jay and Coleman [32].

none of the other three theoretical results shown in Fig. 1(b) show any indication of this feature.

The result of Jay and Coleman [32] is not an absolute cross section but has been normalized to earlier experimental work, so while the present result is about $70 \%$ larger at $3 \mathrm{eV}$, the significance of this is not clear. It may be that some of this difference is due to forward-scattering effects in the data of [32], which are not corrected for in their analysis. The two cross sections are in good agreement above $9 \mathrm{eV}$. Three theoretical results for $\sigma_{\mathrm{GT}}-\sigma_{\mathrm{Ps}}$ from the literature $[2,41,42]$ and the present ROP calculations are also shown in Fig. 1(b). One observation from the results is that both the present experimental and theoretical results do not show any evidence of the Ramsauer-Townsend minimum as predicted by the earliest result of Massey et al. [42] at about $1 \mathrm{eV}$. The
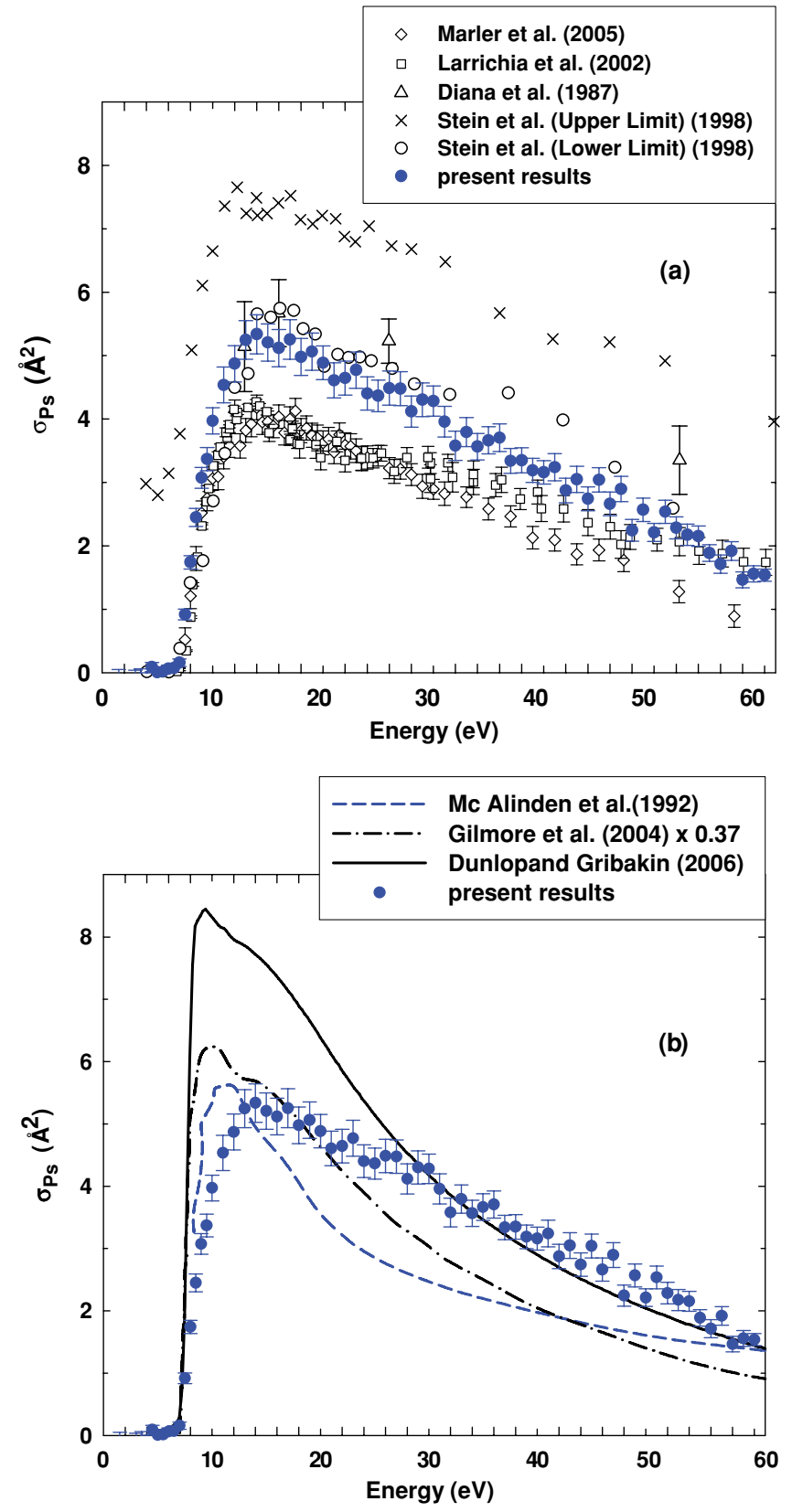

FIG. 2. (Color online) The present absolute total positronium formation cross sections $\sigma_{\mathrm{Ps}}$ compared with (a) previous experimental determinations from Marler et al. [3], Laricchia et al. [10], Diana et al. [43], and Stein et al. [44] and (b) theoretical models from McAlinden and Walters [36], Gilmore et al. [4] and Dunlop and Gribakin [45].

present ROP result shows good qualitative agreement with the previous calculations of Parcell et al. [2] and Schrader [40] below about $15 \mathrm{eV}$. Above this energy qualitative and quantitative differences are observed between the result of [2] and the present ROP cross section, with the ROP result lying in excellent agreement with the present experimental result between 20 and $60 \mathrm{eV}$. However, there are significant differences between the present experimental and ROP results in the region between 1.5 and $20 \mathrm{eV}$, where the experimental cross section is as much as $30 \%$ higher than the theory. 


\section{Ps formation cross section $\sigma_{\mathrm{Ps}}$}

In this section the present experimental results for the total Ps formation cross section for $\mathrm{Kr}$ are presented in Table II and compared with experimental results from the literature in Fig. 2(a) and with theoretical results in Fig. 2(b).

As can be clearly seen in Figs. 2(a) and 2(b), there is a significant spread in the absolute magnitudes of both previous measurements and theoretical results for the Ps formation cross section. This lack of agreement has recently been discussed by Laricchia et al. [10] and Marler et al. [3]. One goal of the present work was to investigate these discrepancies and hopefully improve the accuracy of our knowledge of the $\sigma_{\mathrm{Ps}}$ for $\mathrm{Kr}$. A detailed analysis of the various experimental techniques used for the previous measurements and their strengths and weaknesses has been carried out by both Laricchia et al. and Marler et al. and therefore is not repeated here. The experimental technique used by Laricchia et al. is that of measuring the total ion yield and then subtracting the direct ionization cross section obtained from the literature to reveal the Ps formation cross section. Marler et al. used a technique similar to the present one, where the Ps cross section is measured directly.

Thus, the significant disagreement observed between the present result and that of [3] was a matter of considerable surprise and concern. One possible explanation, however, is that Marler et al. use the single-scattering approximation in deriving $\sigma_{\mathrm{Ps}}$ in their work, whereas the present results are obtained by the use of the Beer-Lambert attenuation law, as has been fully discussed in [18]. To investigate the effect of this, we analyzed the present raw experimental data using the same technique as Marler et al. and this removes most, but by no means all, of the discrepancy seen in Fig. 2(a).

The present $\sigma_{\mathrm{Ps}}$ results are in closer agreement with the experimental results of Diana et al. [43] and the lower limit (LL) results of Stein et al. [44]. The former authors use a method whereby they measure the disappearance of the positrons in the final state (a measure of all Ps formed), while the latter authors measured the simultaneous emission of two $\gamma$ rays coming from singlet (or para-) Ps and quenched triplet (or ortho-) Ps. In principle, the present technique of measuring the loss of positrons is the same as that used by Diana et al., and hence, the qualitative and quantitative agreement is perhaps to be expected. The upper limit results of Stein et al. were measured in the same way as the Diana et al. experiments. However, these are larger than the present result over the whole energy range of overlap, e.g., about $60 \%$ larger at the peak in the cross section at around $15 \mathrm{eV}$. On the contrary, the present result is in reasonable agreement with the LL results of Stein et al., and this would seem to imply that the amount of Ps decay through three-gamma annihilation is negligible.

The present result does show the same energy dependence as the result of Marler et al., albeit being larger in magnitude over the whole energy range of overlap above $E_{\mathrm{Ps}}$, the difference reaching $\sim 40 \%$ at the peak energy of $15 \mathrm{eV}$. It is, however, different from the result of Laricchia et al. at energies above $15 \mathrm{eV}$. One of the differences worth noting is that Laricchia et al. reported a second peak in $\sigma_{\mathrm{Ps}}$ that is not observed in the present result. The origin of this structure has been the subject of some speculation and is discussed in detail by Marler et al. and attributed to possible systematic effects in the different sources of total and direct ionization measurements that were used in [10] to derive their $\sigma_{\mathrm{Ps}}$ results.

In Fig. 2(b) we see generally good agreement in the energy dependence of the Ps formation cross section in the comparison between the present result and the three theoretical results available from the literature $[4,18,36]$, two of which are coupled-channel calculations [4,36] and one of which is a many-body-theory approach [45]. However, there are significant qualitative differences observed. All the theoretical results show a peak in the cross section at about $10 \mathrm{eV}$, whereas the present experimental result peaks at about $15 \mathrm{eV}$. In addition, all three theories predict a larger $\sigma_{\mathrm{Ps}}$ than experiment below $13 \mathrm{eV}$, with the difference being up to $60 \%$ in the case of the calculation of Dunlop and Gribakin [45]. Good agreement, however, is observed between the present results and this calculation at energies above $30 \mathrm{eV}$.

\section{B. Elastic differential cross sections}

The present experimental and theoretical elastic differential cross-section (DCS) results are shown in Figs. 3 and 4 and are also compared with the relatively scarce results available in the published literature. The present theoretical results represent values obtained using both the CCC and the ROP methods.

Figures 3(a)-3(d) show the present elastic DCS results for energies 1, 2, 5, and $8 \mathrm{eV}$, respectively. These are compared with the only other experimental data available in the literature, at 1 and $2 \mathrm{eV}$, from Gilbert et al. [5], whose experimental technique was similar to the present one. In addition to the present calculations, we also show theoretical cross sections by Sin Fai Lam [8] for 1, 2, and $8 \mathrm{eV}$. A common feature of the DCS for all these energies, as indicated in all four panels of Fig. 3, is that they all rise sharply in the forward direction toward the $0^{\circ}$ scattering angle. Again, this is primarily due to the relatively large atomic dipole polarizability of $\mathrm{Kr}$, which results in enhanced long-range interactions and stronger forward-angle scattering at these energies.

The agreement between the two experimental determinations at 1 and $2 \mathrm{eV}$ is excellent, both in magnitude and angular dependence. Subtle differences are observed between the two present theoretical results, below $10^{\circ}$ at 1 and $2 \mathrm{eV}$ and below $40^{\circ}$ at 5 and $8 \mathrm{eV}$. These are thought to be due to differences in the way in which the polarization interaction is treated. The theoretical results of Sin Fai Lam underestimate the amount of forward scattering at all the energies shown in Figs. 3(a)3(d). These results were obtained using the Pople-Schofield approximation for the dipole polarization potential. Our ROP calculations, for example, include the dipole and the next three higher multipole polarization potentials. Increasing the number of multipoles increases the cross section at all angles, compared to the case of using only the dipole. This, in part, may explain some of the differences observed between our results and those of Sin Fai Lam at forward angles. The experimental data for 5 and $8 \mathrm{eV}$ are in better agreement with the CCC results than the ROP, especially in regard to the extent of forward scattering.

Figures 4(a)-4(e) show the present elastic DCS results for energies of $9,10,15,20$, and $30 \mathrm{eV}$, respectively, compared with values from the literature. The results of Kauppila et al. 

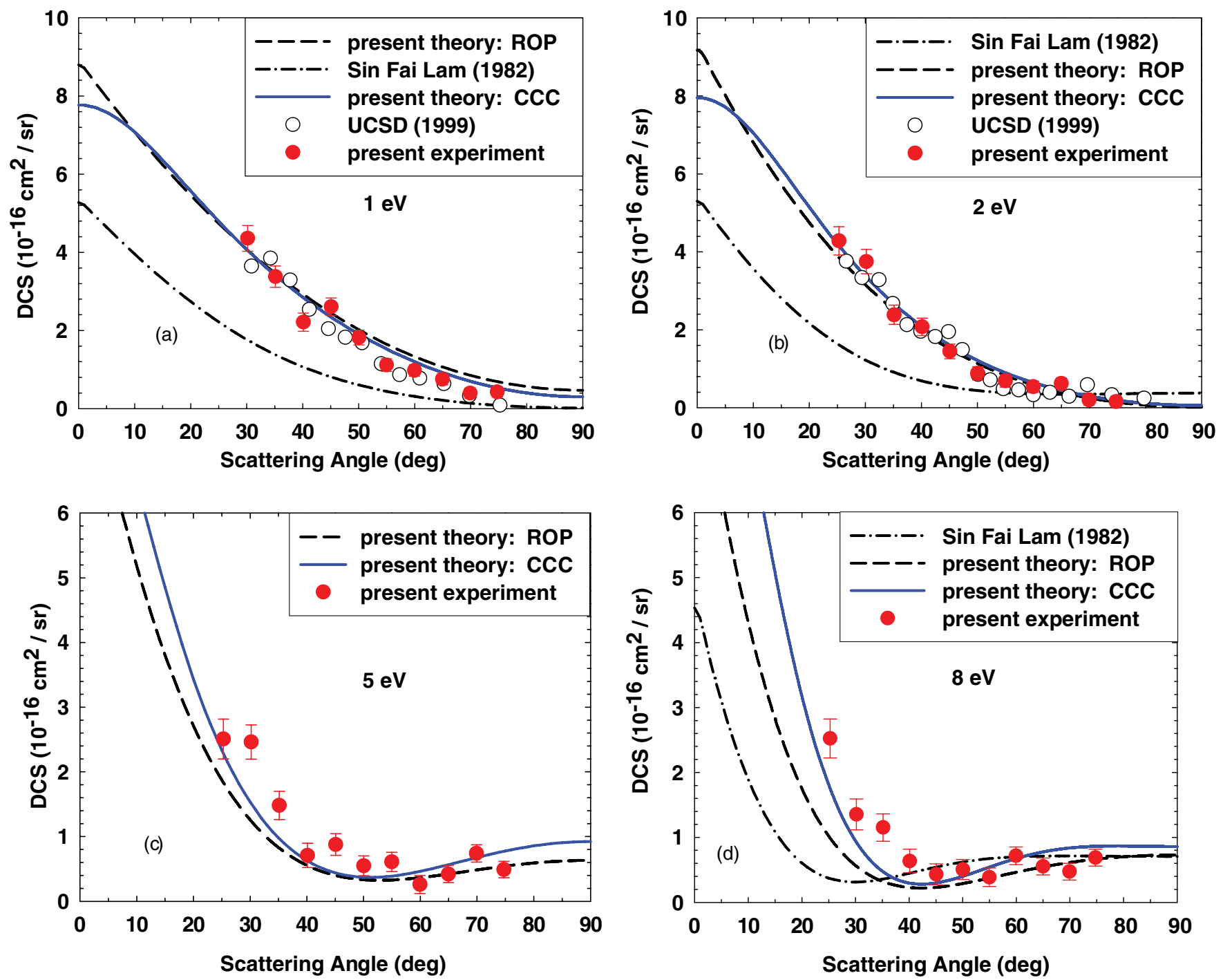

FIG. 3. (Color online) Absolute elastic differential cross sections $\left(10^{-16} \mathrm{~cm}^{2} \mathrm{sr}^{-1}\right)$ for positron scattering from Kr compared with the literature experimental values by the UCSD group [5] and theoretical values from Sin Fai Lam [8] at energies from 1 to $8 \mathrm{eV}$.

[46] presented in Figs. 4(b) and 4(d) for 10 and $20 \mathrm{eV}$, respectively, have been folded about $90^{\circ}$ to enable comparison with the current data (see Sec. II B). In addition, as their values were relative cross sections, they have also been normalized to the present data at $30^{\circ}$. It is worth noting that to obtain the absolute values that were published, Kaupilla et al. normalized their data to the nonrelativistic polarized orbital calculation of [7].

The agreement between the present experimental data and that of [46] is reasonably good over the range of overlap. The results from the three theoretical approximations shown in these panels differ significantly in angular dependence and magnitude at all energies, except at $30 \mathrm{eV}$, where the present CCC and ROP show a similar angular dependence. At this energy, both theoretical curves are also in close agreement with the present experimental values. Overall, the CCC calculation shows the best agreement with the experimental data of the three theoretical approximations. Note that we do not present $\mathrm{CCC}$ results at 9 and $10 \mathrm{eV}$ as this is in the energy region between $E_{\mathrm{Ps}}$ and $E_{\mathrm{ion}}$, where the multicentered approach discussed earlier is clearly required. It is also worth noting that, as shown in Figs. 4(b)-4(d), the results of Sin Fai Lam shows the smallest DCS values at forward angles, below $25^{\circ}$.

\section{CONCLUSION}

In this paper we present absolute experimental measurements of the $\sigma_{\mathrm{GT}}, \sigma_{\mathrm{Ps}}$, and $\sigma_{\mathrm{GT}}-\sigma_{\mathrm{Ps}}$ cross sections for positron scattering from krypton at energies from 0.5 to $60 \mathrm{eV}$. The CCC and ROP approaches have been used to calculate the $\sigma_{\mathrm{GT}}$ and $\sigma_{\mathrm{GT}}-\sigma_{\mathrm{Ps}}$ cross sections, respectively. These data are compared to experimental and theoretical calculations from the literature, for which there existed significant differences for all three cross sections. Absolute measurements of the elastic DCS are also carried out, together with theoretical computations of these cross sections using our two techniques for energies $1,2,5,8$, $9,10,15,20$, and $30 \mathrm{eV}$. 

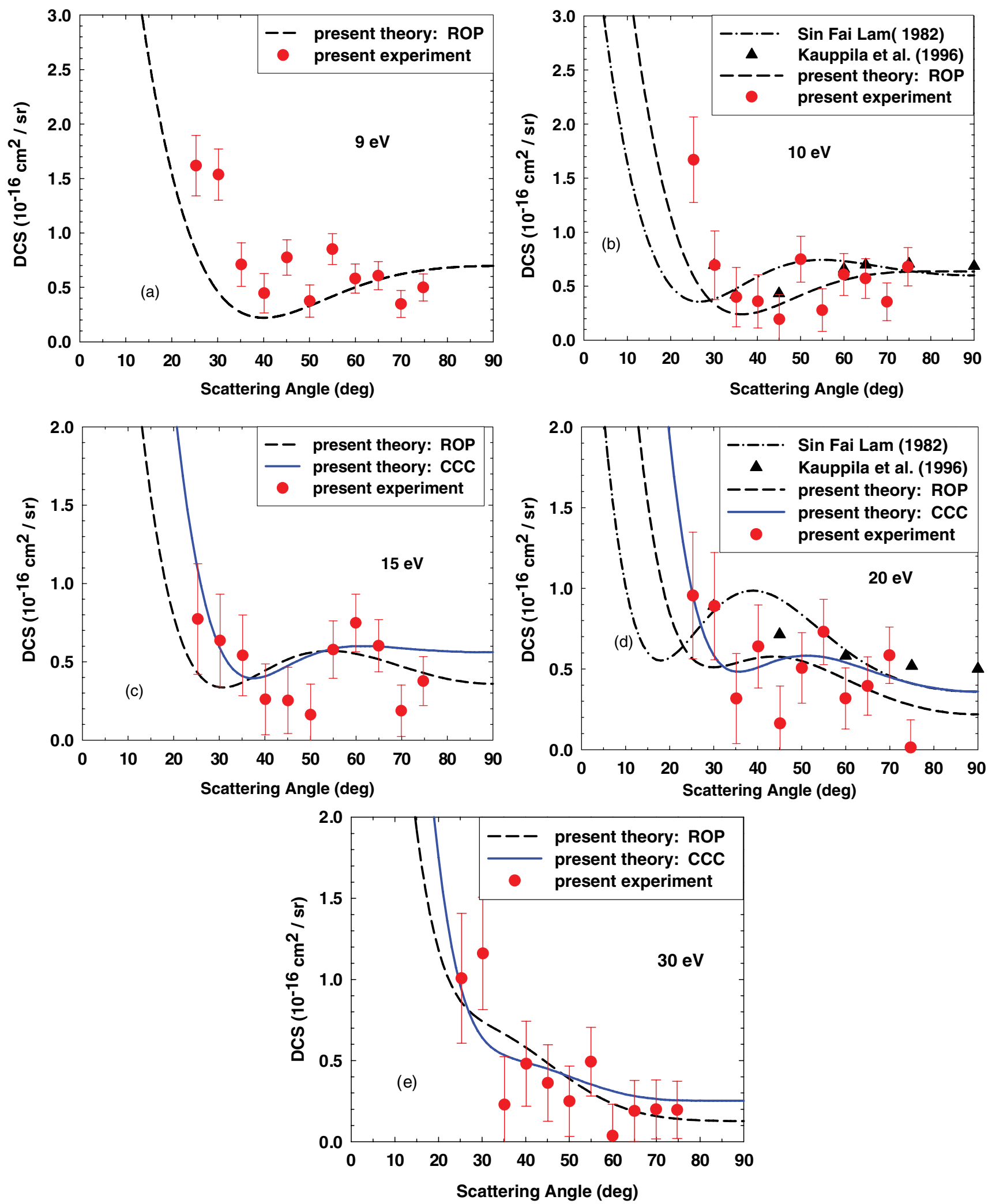

FIG. 4. (Color online) Same as Fig. 3 but for energies from 9 to $30 \mathrm{eV}$ compared with the experimental values of Kauppila et al. [46] and theoretical results of Sin Fai Lam [8].

The present $\sigma_{\mathrm{GT}}$ measurements show good agreement with the present CCC results, except in the region between the thresholds for Ps formation and ionization. Qualitative agreement is also observed with a number of previous results, and good overall agreement is found with the preliminary data of [34], albeit with the present values being higher over all the 
energy range of overlap. Good agreement is observed with the results of [1] at energies above $30 \mathrm{eV}$, while there are significant energy dependence and magnitude differences between the present results and those of [2]. Significant differences are also observed between the present $\sigma_{\mathrm{GT}}-\sigma_{\mathrm{Ps}}$ and most literature values. Agreement with the ROP results for this cross section is good above $20 \mathrm{eV}$, but significant differences prevail between 1.5 and $20 \mathrm{eV}$. The present $\sigma_{\mathrm{Ps}}$ results are in better agreement with the experimental results of [43] and the lower limit results of [44]. Although the same energy dependence is observed compared with the result of [3], the present result is larger in magnitude over the whole energy range of overlap above $E_{\mathrm{Ps}}$, the difference reaching about $40 \%$ at the peak of the cross section at an energy of $15 \mathrm{eV}$. The present result is different from the result of [10] at energies between 10 and $40 \mathrm{eV}$. One of the notable differences being that no second peak is observed in the present cross section at higher energies, as was the case in [10]. The two results, however, are in good agreement at energies above $40 \mathrm{eV}$.

The comprehensive and extensive set of absolute elastic DCS results are in good qualitative and quantitative agreement with the experimental results of [5] at the common energies of 1 and $2 \mathrm{eV}$. A comparison with the results of [6], at the common energies of 10 and $20 \mathrm{eV}$, also yields good agreement in the angular dependence. Both of the present calculations show excellent agreement with experiment at low incident energies. At higher energies the level of agreement is not quite so good with the CCC calculation, perhaps showing the better overall agreement with the present experimental values. The theoretical approximation of [8] appears to underestimate the extent of forward scattering at angles below $30^{\circ}$ at all the energies for which results are available.

Overall, there is a substantial body of work for lowenergy positron scattering from $\mathrm{Kr}$, and this contribution, we hope, adds to that by providing more extensive, absolute measurements of differential scattering and total scattering cross sections in which forward-scattering effects have been carefully accounted for. Outstanding differences of the order of $20 \%$ remain for the magnitude of the Ps formation cross section in the energy range between 10 and $40 \mathrm{eV}$.

\section{ACKNOWLEDGMENTS}

The authors gratefully acknowledge the Australian Research Council's (ARC) Centre of Excellence Program for providing funding. One of us (C.M.) is also grateful to the ARC for financial support under the Australian Postdoctoral Fellowships program, and M.H. thanks the Researcher Exchange Program between the Japan Society for the Promotion of Science and the Australian Academy of Science for a grant to visit the ANU and Flinders University. It is a pleasure to thank Antonio Zecca and Michael Brunger for useful discussions and for providing their unpublished data. We would also like to thank the technical staff at the ANU, Graeme Cornish, Stephen Battisson, Ross Tranter, and Ron Cruikshank for their invaluable input.
[1] K. L. Baluja and A. Jain, Phys. Rev. A 46, 1279 (1992).

[2] L. A. Parcell, R. P. McEachran, and A. D. Stauffer, Nucl. Instrum. Methods. Phys. Res. Sect. B 171, 113 (2000).

[3] J. P. Marler, J. P. Sullivan, and C. M. Surko, Phys. Rev. A 71, 022701 (2005).

[4] S. Gilmore, J. E. Blackwood, and H. R. J. Walters, Nucl. Instrum. Methods. Phys. Res., Sect. B 221, 129 (2004)

[5] S. J. Gilbert, R. G. Greaves, and C. M. Surko, Phys. Rev. Lett. 82, 5032 (1999).

[6] W. E. Kaupilla, C. K. Kwan, D. Przybyla, S. J. Smith, and T. S. Stein, Can. J. Phys. 74, 474 (1996).

[7] R. P. McEachran, A. D. Stauffer, and L. E. M. Campbell, J. Phys. B 13, 1281 (1980).

[8] L. T. Sin Fai Lam, J. Phys. B 15, 143 (1982).

[9] A. D. Stauffer, L. A. Parcell, and R. P. McEachran, Nucl. Instrum. Methods. Phys. Res. Sect. B 221, 93 (2004).

[10] G. Laricchia, P. Van Reeth, M. Szluinska, and J. Moxom, J. Phys. B 35, 2525 (2002).

[11] K. Bartschat, Phys. Rev. A 71, 032718 (2005).

[12] R. P. McEachran and A. D. Stauffer, J. Phys. B 43, 215209 (2010).

[13] G. Sinapius, W. Raith, and W. G. Wilson, J. Phys. B 13, 4079 (1980).

[14] T. J. Murphy and C. M. Surko, Phys. Rev. A 46, 5696 (1992).

[15] S. J. Gilbert, C. Kurz, R. G. Greaves, and C. M. Surko, Appl. Phys. Lett. 70, 1944 (1997).

[16] J. P. Sullivan, A. Jones, P. Caradonna, C. Makochekanwa, and S. J. Buckman, Rev. Sci. Intrum. 79, 113105 (2008).
[17] J. P. Sullivan, S. J. Gilbert, J. P. Marler, R. G. Greaves, S. J. Buckman, and C. M. Surko, Phys. Rev. A 66, 042708 (2002).

[18] A. C. L. Jones et al., Phys. Rev. A 83, 032701 (2010).

[19] J. P. Sullivan, C. Makochekanwa, A. Jones, P. Caradonna, D. S. Slaughter, J. Machacek, R. P. McEachran, D. W. Mueller, and S. J. Buckman, J. Phys. B 41, 081001 (2008).

[20] J. P. Sullivan, C. Makochekanwa, A. Jones, P. Caradonna, D. S. Slaughter, J. R. Machacek, R. P. McEachran, D. W. Mueller, and S. J. Buckman, J. Phys. B 44, 035201 (2011).

[21] J. P. Sullivan, A. Jones, P. Caradonna, C. Makochekanwa, and S. J. Buckman, Nucl. Instrum. Methods Phys. Res. Sect. B 266, 384 (2008).

[22] P. Caradonna, J. P. Sullivan, A. Jones, C. Makochekanwa, D. S. Slaughter, D. W. Mueller, and S. J. Buckman, Phys. Rev. A 80, 060701(R) (2009).

[23] S. Chen, R. P. McEachran, and A. D. Stauffer, J. Phys. B 41, 025201 (2008).

[24] R. Utamuratov, A. S. Kadyrov, D. V. Fursa, I. Bray, and A. T. Stelbovics, J. Phys. B 43, 125203 (2010).

[25] I. P. Grant, B. J. McKenzie, P. H. Norrington, D. F. Mayers, and N. C. Pyper, Comput. Phys. Commun. 21, 207 (1980).

[26] I. Bray and A. T. Stelbovics, Phys. Rev. A 46, 6995 (1992).

[27] I. Bray and A. T. Stelbovics, Phys. Rev. A 48, 4787 (1993).

[28] H. Wu, I. Bray, D. V. Fursa, and A. T. Stelbovics, J. Phys. B 37, L1 (2004).

[29] D. V. Fursa and I. Bray, J. Phys. B 30, 5895 (1997).

[30] A. Kumar and W. Meath, Can. J. Chem. 63, 1616 (1985). 
[31] K. F. Canter, P. G. Coleman, T. C. Griffith, and G. R. Heyland, J. Phys. B 6, L201 (1973).

[32] P. M. Jay and P. G. Coleman, Phys. Rev. A 82, 012701 (2010).

[33] M. S. Dababneh, W. E. Kauppila, J. P. Downing, F. Laperriere, V. Pol, J. H. Smart, and T. S. Stein, Phys. Rev. A 22, 1872 (1980).

[34] A. Zecca, L. Chiari, E. Trainotti, and M. J. Brunger (private communication, 2010).

[35] A. Zecca, L. Chiari, E. Trainotti, A. Sarkar, and M. J. Brunger, PMC Phys. B 3, 4 (2010).

[36] M. T. McAlinden and H. R. J. Walters, Hyperfine Interact. 73, 65 (1992).

[37] R. I. Campeanu, R. P. McEachran, and A. D. Stauffer, Can. J. Phys. 74, 544 (1996).

[38] R. I. Campeanu, R. P. McEachran, and A. D. Stauffer, Can. J. Phys. 77, 769 (1999).

[39] R. I. Campeanu, R. P. McEachran, and A. D. Stauffer, Nucl. Instrum. Methods Phys. Res. Sect. B 192146 (2002).
[40] A. C. L. Jones, P. Caradonna, C. Makochekanwa, D. S. Slaughter, R. P. McEachran, J. R. Machacek, J. P. Sullivan, and S. J. Buckman, Phys. Rev. Lett. 105, 073201 (2010).

[41] D. M. Schrader, Phys. Rev. A 20, 918 (1979).

[42] H. S. W. Massey, J. Lawson, and D. G. Thompson, Quantum Theory of Atoms, Molecules, Solid State (Academic, New York, 1966), p. 203.

[43] L. M. Diana, P. G. Coleman, D. L. Brooks, and R. L. Chaplin, Atomic Physics with Positrons, edited by J. W. Humberston and E. A. G. Armour (Plenum, New York, 1987), p. 55.

[44] T. S. Stein, M. Harte, J. Jiang, W. E. Kauppila, C. K. Kwan, H. Li, and S. Zhou, Nucl. Instrum. Methods Phys. Res. Sect. B 143, 68 (1998).

[45] L. J. M. Dunlop and G. F. Gribakin, Nucl. Instrum. Methods Phys. Res. Sect. B 247, 61 (2006).

[46] W. E. Kauppila, C. K. Kwan, D. Przybyla, S. J. Smith, and T. S. Stein Can. J. Phys. 74, 474 (1996). 